\title{
Facing the future: face-emotion processing deficits as a potential biomarker for various psychiatric and neurological disorders
}

\begin{abstract}
Linda Isaac*
Clinical Psychology, Behavioural Science Institute, Radboud University, Nijmegen, Netherlands

*Correspondence: l.isaac@psych.ru.nl
\end{abstract}

\section{A commentary on}

A comparison of facial emotion processing in neurological and psychiatric conditions by Bediou, B., Brunelin, J., d'Amato, T., Fecteau, S., Saoud, M., Hénaff, M., and Salmon, P. K. (2012). Front. Psychol. 3:98. doi: 10.3389/fpsyg.2012.00098

This commentary highlights key points presented by Bediou et al. (2012) in Frontiers in Psychology. The authors have provided a foundation upon which to investigate face-emotion processing as a biomarker for both psychological and neurological diseases. To date, Bediou et al. (2012) are the first to provide a comprehensive analysis of the unique deficits in face-emotion processing associated with a wide range of diseases including but not limited to neurodegenerative diseases, mood disorders, psychopathology, and neurodevelopment disorders.

Recently there has been considerable interest in face-related research spanning various disciplines from psychology, neurology, and computational science. Moreover, face-emotion processing research has taken center stage in affective neuroscience leading researchers to investigate the complex relationship between disease, brain, cognition, behavior, and emotion as it relates to impairments in the processing of human faces at various stages of structural encoding, identification and familiarity, emotion recognition, and semantic retrieval pertaining to specific known faces. Bediou et al. (2012) present data from their previous work on face processing drawing from:

- Parkinson's disease,

- fronto-temporal dementia,

- mild cognitive impairment,

- Alzheimer's disease,

- major depression, and schizophrenia.
In our research, we have detected mood-congruent attentional biases for sad and angry faces in experimentally induced sadness (Isaac et al., 2012). This supports the hypothesis that human faces do indeed have "privileged access" to the affective network even at the transient phase of experimentally putting healthy participants into a sad mood state (De Houwer and Hermans, 1994). Additionally, Bediou et al. (2012) focused upon additional groups of interest for risk evaluation such as unaffected siblings of Schizophrenia patients and present the means by which medication status may modulate processing of valenced faces. This latter point is neglected too often and it remains especially important to consider due to the role of antidepressant treatment in modulating amygdala response to positive or happy faces (Norbury et al., 2009).

The objective of this commentary is to discuss the implications of Bediou et al.'s (2012) article. In summary, they conclude that facial emotion processing is indeed impaired in subjects exhibiting a variety of both psychiatric and neurological disorders. The faceemotion impairments appear to be both characteristic and specific to each disease. One can speculate that both the prevalence and specificity of face processing differences across such diseases can be employed as a potential biomarker that may be used to perform differential diagnoses, treatment outcome measurements as well as treatment response predictions. Unquestionably, this idea is bound to stimulate debate among researchers and clinicians alike. Much effort has gone into the neural network of faceemotion processing (Kanwisher et al., 1997; Haxby et al., 2000), face-specific neurophysiology (Bentin et al., 1996), computational modeling of face processing (Wallraven et al., 2005), the mechanics that underlie experience dependent face processing such as featural vs. configural strategies (Yin, 1969), and the developmental process of face expertise (Gauthier and Nelson, 2001). Notably, face-emotion processing deficit extends to populations beyond those studied by Bediou et al. (2012). Advancements in face and emotion processing science have resulted in specific neurophysiological markers such as the P100 (initial visual processing of faces), N170 (structural encoding of facial features), N250 (decoding emotional content), and N450 (semantic retrieval for familiar faces). Impaired face-emotion processing extends to other pathologies such as social anxiety (Lange et al., 2011), Autism (Pierce et al., 2001), and Williams Syndrome: a rare genetic disorder (Isaac and Lincoln, 2011) as well as traumatic brain injury (Valentine et al., 2006).

Interestingly, the various deficits often implicate specific brain regions as in lesion to the fusiform gyri and facial agnosia (Behrmann et al., 1994) or an enlarged amygdala and attenuated response to angry faces in Williams Syndrome (Golarai et al., 2010) and thus the connection is made between brain, behavior, and emotion stemming from faulty face processing. Thanks to the outstanding research and clinical advancements in this field, high prevalence, and relevance of face-emotion anomalies in a vast number of special populations has been established. It is proposed that face processing deficits be employed as both a biomarker for differential diagnosis and as a treatment outcome measurement. Furthermore, it is suggested that treatment response prediction shall become the ultimate goal of face-emotion science.

The question is where do we go from here? The next lines of enquiry may ask the following questions:

- How can specific face-emotion deficits aid in differential diagnosis?

- How can face-specific deficits inform us about treatment outcome and treatment response? 
Such efforts are already underway. For example, studies examining the effectiveness of modifying facial cognitive biases in anxiety disorders have demonstrated that re-training such biases and improving cognitive control reduces anxiety level (MacLeod et al., 2002; Wadlinger and Isaacowitz, 2008). Do certain patients suffer from temporal deficits concerning emotional faces despite successful treatment as is the case of remitted depression and sustained working memory disengagement deficits for angry faces (Isaac et al., submitted)? Do patients activate control centers more variably over time compared to non-patients? Is it a matter of spatial magnitude whereby patients activate control centers to a lesser degree or more diffusely than controls (e.g., PFC/ ACC)? What vulnerabilities exist at the prodromal stage of disease that can possibly be ameliorated? Importantly, how can this research guide the clinical process to improve patient quality of life?

For instance, in schizophrenia research, we are now armed with the knowledge that abnormalities in late-stage feature decoding exist and can potentially underlie emotion identification deficits in this disorder (Wynn et al., 2008). This indeed is a potential biomarker of underlying facial affect deficits in schizophrenia and it may very well be associated with the social cognitive deficits observed in schizophrenia. However, questions remain as to how facial-decoding abnormalities can explain clinical features such as paranoia and how this information can facilitate the development of new treatments that enhance social functioning in schizophrenia, depression, and other patient populations that may benefit from improved face-emotion processing. To bring attention to this field, I have compiled a collection of outstanding international research contributions in a special issue entitled, "Face Processing: Perspectives from Cognitive Science and Psychopathology" (Frontiers in Cognitive Science).

\section{REFERENCES}

Bediou, B., Brunelin, J., d'Amato, T., Fecteau, S., Saoud, M., Hénaff, M., and Salmon, P. K. (2012). A comparison of facial emotion processing in neurological and psychiatric conditions. Front. Psychol. 3:98. doi: 10.3389/fpsyg.2012.00098

Behrmann, M., Moscovitch, M., and Winocur, G. (1994). Intact visual imagery and impaired visual perception in a patient with visual agnosia. J. Exp. Psychol. Hum. Percept. Perform. 20, 1068-1087.

Bentin, S., Allison, T., Puce, A., Perez, E., and McCarthy, G. (1996). Electrophysiological studies of face perception in humans. J. Cogn. Neurosci. 8, 551-565.

De Houwer, J., and Hermans, D. (1994). Differences in the affective processing of words and pictures. Cogn. Emot. 8, 1-20.

Gauthier, I., and Nelson, C. (2001). The development of face expertise. Curr. Opin. Neurobiol. 11,219-224.

Golarai, G., Hong, S., Haas, B. W., Galaburda, A. M., Mills, D. L., Bellugi, U., Grill-Spector, K., and Reiss, A. L. (2010). The fusiform face area is enlarged in Williams syndrome. J. Neurosci. 30, 6700-6712.

Haxby, J. V., Hoffman, E. A., and Gobbini, I. M. (2000). The distributed human neural system for face perception. Trends Cogn. Sci. (Regul. Ed.) 4, 223-233.

Isaac, L., and Lincoln, A. (2011). Featural versus configural face processing in a rare genetic disorder: Williams syndrome. J. Intellect. Disabil. Res. 55, 1034-1042.

Isaac, L., Vrijsen, J.N., Eling, P., van Oostrom, I., Speckens, A., and Becker, E. S. (2012). Verbal and facial-emotional Stroop tasks reveal specific attentional interferences in sad mood. Brain Behav. 2, 74-83.

Kanwisher, N. G., McDermott, J., and Chun, M. M. (1997). The fusiform face area: a module in human extrastriate cortex specialized for face perception. $J$. Neurosci. 17, 4302-4311.

Lange, W. G., Heuer, K., Langner, O., Keijsers, G. P. J., Becker, E. S., and Rinck, M. (2011). Face value: eye movements and the evaluation of facial crowds in social anxiety. J. Behav. Ther. Exp. Psychiatry 42, 355-363.

MacLeod, C., Rutherford, E., Campbell, L., Ebsworthy, G., and Holker, L. (2002). Selective attention and emotional vulnerability: assessing the causal basis of their association through the experimental manipulation of attentional bias. J. Abnorm. Psychol. 111, 107-123.

Norbury, R., Taylor, M. J., Selvaraj, S., Murphy, S. E., Harmer, C. J., and Cowen, P. J. (2009). Short-term antidepressant treatment modulates amygdala response to happy faces. Psychopharmacology (Berl.) 206, 197-204

Pierce, K., Müller, R. A., Ambrose, J., Allen, G., and Courchesne, E. (2001). Face processing occurs outside the fusiform "face area" in autism: evidence from functional MRI. Brain 124, 2059-2073.

Valentine, T. R., Powell, J. H., Davidoff, J. B., Letson, S., and Greenwood, R. (2006). Prevalence and correlates of face recognition impairments after acquired brain injury. Neuropsychol. Rehabil. 16, 272-297.

Wadlinger, H., and Isaacowitz, D. (2008). Looking happy: the experimental manipulation of a positive visual attention bias. Emotion 8, 121-126.

Wallraven, C., Schwaninger, A., and Bülthoff, H. H. (2005). Learning from humans: computational modeling of face recognition. Network 16, 401-418.

Wynn, J. K., Lee, J., Horan, W. P., and Green, M. F. (2008). Using event related potentials to explore stages of facial affect recognition deficits in schizophrenia. Schizophr. Bull. 34, 679-687.

Yin, R. K. (1969). Looking at upside-down faces. J. Exp. Psychol. 81, 141-145.

Received: 22 April 2012; accepted: 11 May 2012; published online: 12 June 2012.

Citation: Isaac L (2012) Facing the future: face-emotion processing deficits as a potential biomarker for various psychiatric and neurological disorders. Front. Psychology 3:171. doi: 10.3389/fpsyg.2012.00171

This article was submitted to Frontiers in Cognitive Science, a specialty of Frontiers in Psychology.

Copyright $\odot 2012$ Isaac. This is an open-access article distributed under the terms of the Creative Commons Attribution Non Commercial License, which permits non-commercial use, distribution, and reproduction in other forums, provided the original authors and source are credited. 